\title{
Comunicação bucossinusal em região de primeiro pré-molar superior - relato de caso atípico
}

\author{
Buccosinusal communication in the region of the upper first premolar - atypical case report \\ Comunicación bucosinusal en la región del primer premolar superior - reporte de caso atípico
}

Recebido: 08/12/2021 | Revisado: 13/12/2021 | Aceito: 05/01/2022 | Publicado: 08/01/2022

\author{
Luiz Felipe Rocha Vilaça \\ ORCID: https://orcid.org/0000-0001-8619-5205 \\ Universidade Federal de Goiás, Brasil \\ E-mail: vilacaluizfelipe@gmail.com \\ Matheus Pereira Marques \\ ORCID: https://orcid.org/0000-0001-8399-161X \\ Universidade Federal de Goiás, Brasil \\ E-mail: matheuspmrq@gmail.com \\ Robson Rodrigues Garcia \\ ORCID: https://orcid.org/0000-0003-3479-6097 \\ Universidade Federal de Goiás, Brasil \\ E-mail: robsongarcia@ufg.br \\ Rhonan Ferreira da Silva \\ ORCID: https://orcid.org/0000-0002-3680-7020 \\ Universidade Federal de Goiás, Brasil \\ E-mail: rhonan@ufg.br
}

\begin{abstract}
Resumo
Introdução: A comunicação bucossinusal (oroantral) é um acidente decorrente de cirurgias de retirada de lesões, instalação de implantes e extrações dentais que pode resultar em desordens locais, pela comunicação entre o seio maxilar e a cavidade oral. O diagnóstico é realizado pela manobra de Valsalva, exames imaginológicos ou queixa do paciente no pós-operatório. O tratamento indicado depende da extensão, grau de complexidade e condição do paciente. Dentre as alternativas de tratamento está a rotação ou deslizamento de retalho tecidual para a região do alvéolo. A incidência das comunicações bucossinusais é maior na região de molares pela expansão do seio maxilar nessa região. Assim, as comunicações em região de pré-molares, especialmente na região de primeiro pré-molar superior, são atípicas. Objetivo: O presente trabalho tem por objetivo relatar uma comunicação bucossinusal atípica diagnosticada logo após a extração de um primeiro pré-molar. Relato do caso: Paciente C.F.S, do gênero masculino, de 39 anos, foi submetido à exodontia do 14 após fratura radicular (CID 10 - K02.4). No transcirúrgico foi diagnosticada, pela manobra de Valsalva, a comunicação entre o seio maxilar e o alvéolo remanescente. $O$ paciente foi tratado com retalho palatino para preservação do fundo de vestíbulo para tratamento reabilitador protético, sem intercorrências na recuperação. Conclusão: É necessário que o profissional esteja atento às exodontias dos dentes superiores próximos ao seio maxilar, sobretudo, aos pré-molares, embora seja menos comum o envolvimento desses dentes na comunicação bucossinusal.
\end{abstract}

Palavras-chave: Fístula bucoantral; Seio maxilar; Cirurgia bucal; Dente pré-molar.

\begin{abstract}
Introduction: The oroantral (oroantral) communication is an accident resulting from surgery to remove lesions, installation of implants and dental extractions that can result in local disorders, due to the communication between the maxillary sinus and the oral cavity. Diagnosis is made by Valsalva maneuver, imaging exams or the patient's postoperative complaint. The indicated treatment depends on the extension, degree of complexity and condition of the patient. Among the treatment alternatives is the rotation or sliding of the tissue flap to the alveolar region. The incidence of oroantral communications is greater in the molar region due to the expansion of the maxillary sinus in this region. Thus, communications in the premolar region, especially in the upper first premolar region, are atypical. Objective: The present work aims to report an atypical oroantral communication diagnosed soon after the extraction of a first premolar. Case report: Patient C.F.S, male, 39 years old, underwent extraction of 14 after root fracture (CID 10 - K02.4). During surgery, the communication between the maxillary sinus and the remaining alveolus was diagnosed using the Valsalva maneuver. The patient was treated with a palatal flap to preserve the vestibule fundus for a prosthetic rehabilitation treatment, without complications during recovery. Conclusion: It is necessary for the professional to be aware of the extraction of upper teeth close to the maxillary sinus, especially the premolars, although the involvement of these teeth in the oral-sinusal communication is less common.
\end{abstract}

Keywords: Oral fistula; Maxillary sinus; Oral surgery; Bicuspid. 


\begin{abstract}
Resumen
Introducción: La comunicación oroantral (oroantral) es un accidente resultante de la cirugía para extirpar lesiones, instalación de implantes y extracciones dentales que pueden resultar en alteraciones locales, debido a la comunicación entre el seno maxilar y la cavidad bucal. El diagnóstico se realiza mediante maniobra de Valsalva, exámenes de imagen o la queja postoperatoria del paciente. El tratamiento indicado depende de la extensión, grado de complejidad y estado del paciente. Entre las alternativas de tratamiento se encuentra la rotación o deslizamiento del colgajo de tejido hacia la región alveolar. La incidencia de comunicaciones oroantrales es mayor en la región molar debido a la expansión del seno maxilar en esta región. Por tanto, las comunicaciones en la región de los premolares, especialmente en la región del primer premolar superior, son atípicas. Objetivo: El presente trabajo tiene como objetivo reportar una comunicación oroantral atípica diagnosticada poco después de la extracción de un primer premolar. Caso clínico: Paciente C.F.S, varón, 39 años, sometido 14 extracción tras fractura radicular (CIE 10 K02.4). Durante la cirugía se diagnosticó la comunicación entre el seno maxilar y el alvéolo remanente mediante la maniobra de Valsalva. El paciente fue tratado con un colgajo palatino para preservar el fondo de vestíbulo para un tratamiento de rehabilitación protésica, sin complicaciones durante la recuperación. Conclusión: Es necesario que el profesional esté atento a la extracción de dientes superiores cercanos al seno maxilar, especialmente los premolares, aunque la afectación de estos dientes en la comunicación oral-sinusal es menos común.
\end{abstract}

Palabras clave: Fístula oral; Seno maxilar; Cirugía bucal; Diente premolar.

\title{
1. Introdução
}

O termo seio ou antro é derivado do latim de sinus, que significa "estrutura cavitária vazia". No crânio, os seios paranasais são cavidades presentes no interior de quatro ossos (frontal, etmoide, esfenoide e maxilas) e que desempenham funções importantes para o crânio, como proteção mecânica e biológica ao organismo com a produção de muco contra agentes infecciosos (Batista et al. 2011), além de aquecer o ar e participar da ressonância da voz. Em específico, o seio maxilar possui forma piramidal quadrangular com o assoalho condizente com o processo alveolar da maxila com a presença de feixes neurovasculares dos dentes posteriores (Cardoso et al. 2002).

Em decorrência da proximidade do seio maxilar com o ápice dos molares e pré-molares, a realização de procedimentos cirúrgicos próximos a esta região pode resultar em acidentes que provocam desordens locais e comunicação de cavidades distintas. A realização de procedimentos de extração de dentes com raízes divergentes em região posterior de maxila pode resultar na remoção de uma porção óssea do assoalho do seio, principalmente, em processos alveolares pneumatizados em decorrência de perdas dentais antigas (Batista et al. 2011).

A comunicação bucossinusal, também chamada de oroantral, é um acesso indesejado formado pelo contato direto entre o seio maxilar e a cavidade bucal que surge em decorrência de traumas, tumores, retirada de lesões, osteomielite, radioterapia, instalação de implantes e extrações dentais na região póstero-superior de maxila (Camarini et al. 2008) (Palhano et al. 2020), (Macedo et al. 2020), (Rodrigues et al. 2021), (Amorim et al. 2020). Quando não há diagnóstico e tratamento precoce da desordem, há a formação natural de um revestimento de epitelial, passando a chamar-se de fístula bucossinusal (Del Junco et al. 1988), (Yih et al. 1988).

Segundo (Hupp et al. 2008), as sequelas mais preocupantes dessa comunicação provêm do refluxo de saliva e restos alimentares para a cavidade sinusal causando contato direto de bactérias presentes na boca com o seio maxilar e, consequentemente, sinusite pós-operatória. É estimado que cerca de 50\% dos pacientes desenvolvem sinusite pós-operatória após 48 horas da comunicação bucossinusal, enquanto o índice pode chegar a $90 \%$ ao longo de 14 dias sem intervenção (Haas et al. 2003).

O diagnóstico dessa condição em extrações dentais pode ser feito de diversas formas. A primeira delas é a observação pelo Cirurgião-dentista da condição das raízes após a exérese dental com possível remanescente do assoalho da cavidade do seio maxilar. Em seguida, pode ser feito o diagnóstico da comunicação, também, através da manobra de Valsalva que consiste em obstruir a passagem de ar pelo nariz verificando a formação de bolhas no interior do alvéolo durante a expiração do ar (Hupp et al. 2008). 
Há na literatura diversas alternativas de fechamento de comunicação bucossinusal dependendo da extensão, grau de complexidade e condição da região (Amorim et al. 2020), (Macedo et al. 2020). O tratamento mais conservador se restringe às comunicações de 1-2mm que tem fechamento espontâneo. Também, o tratamento de eleição pode variar entre utilização do corpo adiposo bucal, sutura em forma de oito, tratamento medicamentoso, rotação de retalho palatino, retalho deslizante vestibular ou enxertos ósseos (Scattarella et al. 2010).

Nesse sentido, a prevalência de comunicações bucossinusais em molares é bem maior que em pré-molares. Estima-se que a incidência gira em torno de $30 \%$ de casos em terceiro molar, $45 \%$ em segundo molar e 27,2\% em primeiros molares. Essa relação fica ainda menor quando se tange aos primeiros pré-molares que apresentam índices de apenas 5,3\% de todos os casos de comunicação oroantral (Parvini et al. 2019).

Devido aos poucos relatos presentes na literatura sobre a incidência desse tipo de complicação incluindo, em especial, o primeiro pré-molar, o presente caso discute que o adequado diagnóstico clínico e planejamento cirúrgico são indispensáveis, pois uma intercorrência como essa, embora rara, é possível. Então, esse trabalho tem por objetivo relatar uma comunicação bucossinusal atípica diagnosticada logo após a extração de um primeiro pré-molar.

\section{Metodologia}

O presente trabalho trata-se de um relato de caso no formato descritivo e qualitativo sobre o tratamento de uma intercorrência pouco comum em exodontia de primeiros pré-molares superiores. Sendo assim, caracteriza-se como uma pesquisa que, por via direta, recolheu dados relativos ao estudo por meio da anamnese, histórico médico e odontológico, bem como, registros fotográficos coletados e autorizados por meio do prontuário do paciente, junto ao Termo de Consentimento Livre e Esclarecido (TCLE) (Pereira et al. 2018). Para embasamento teórico foram feitas consulta de dados nos bancos: Scientific Electronic Library Online (Scielo), National Library of Medicine (PubMed), Google Acadêmico e livros de referência.

\section{Relato do Caso}

Paciente C.F.S, do gênero masculino, 39 anos, compareceu à Clínica de Urgência da Faculdade de Odontologia da Universidade Federal de Goiás (FO-UFG) com queixa de dor no dente 14. Após a realização de exames clínicos e radiográficos, a conduta foi a abertura coronária, instrumentação e inserção de medicação intracanal. O procedimento foi realizado sem intercorrências. O paciente não possuía nenhum comprometimento sistêmico e não fazia tratamento médico.

No $13^{\circ}$ dia de medicação intracanal, no entanto, o paciente retornou à Clínica de Urgência com queixa de fratura coronal. O exame clínico constatou fratura da cúspide palatal. Na tentativa de manter o dente e prosseguir com um tratamento conservador, optou-se pela realização da cirurgia periodontal com o objetivo de aumentar a coroa clínica do dente em questão. Durante o transoperatório, contudo, foi observada uma linha de fratura vertical que envolvia a raiz do referido dente (Figura 1). A partir dessa intercorrência, que inviabilizou a continuidade do tratamento conservador, a exodontia foi indicada. 
Figura 1. Radiografia periapical pré-operatória, evidenciando a pneumatização do processo alveolar e a proximidade do seio maxilar com o periápice do dente 14 .

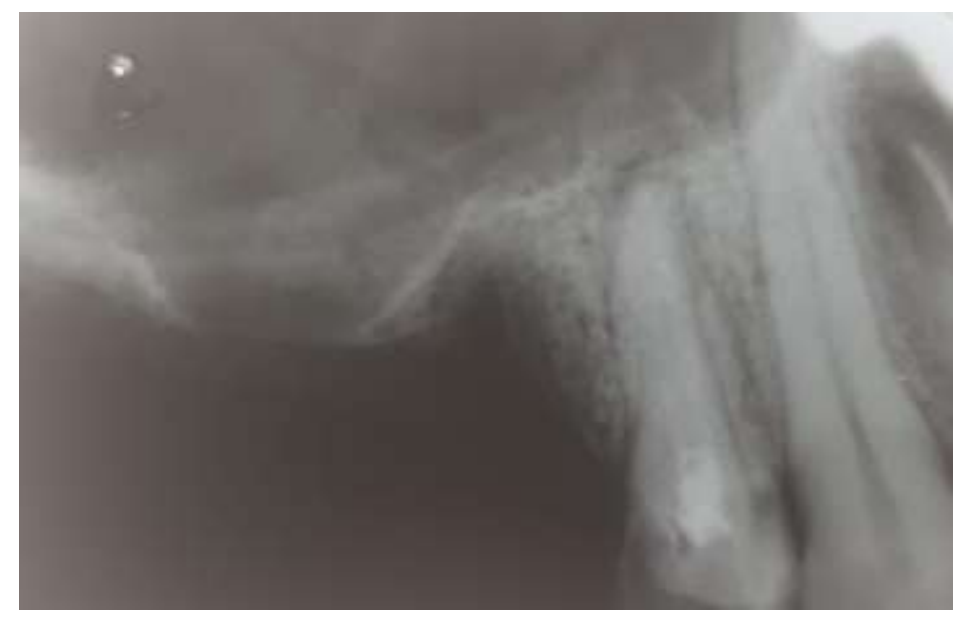

Fonte: Autores (2022).

A exodontia do dente 14 foi realizada em nível ambulatorial, sob anestesia local. Foi utilizado Lidocaína $2 \%$ 1:100.000 de epinefrina, e, como técnicas anestésicas, foram feitos bloqueios dos nervos alveolar superior posterior, alveolar superior médio e palatino maior. Em seguida, incisão intrasulcular com lâmina fria número 15, sindesmotomia dos tecidos com descolador de Molt, luxação com alavancas Seldin Reta e Apexo, e fórceps 150 para tração e remoção do dente. Durante o transcirúrgico houve a fratura da raiz palatal, com posterior remoção (Figura 2). Ao final, como conduta padrão, foi feita a Manobra de Valsalva, e nesse momento, associado a critérios clínicos, como a inspeção e o diagnóstico radiográfico, foi diagnosticada a comunicação bucossinusal imediata.

Figura 2. Dente 14 removido com raiz palatal fraturada.

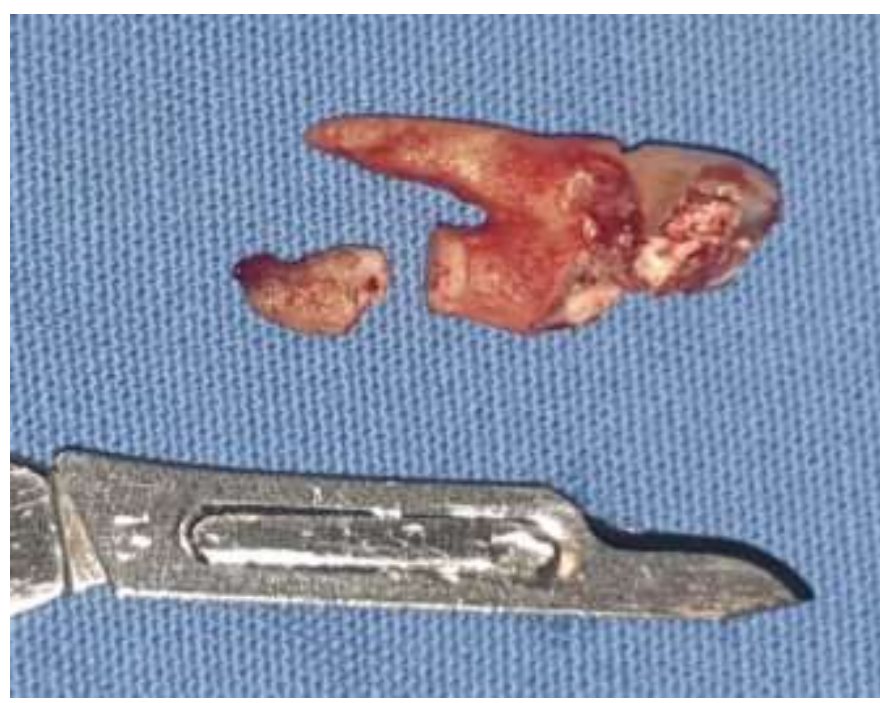

Fonte: Autores (2022).

Como modalidade de tratamento, elegeu-se a rotação do retalho palatino para a região tratada uma vez que a abertura superior do alvéolo remanescente estava mais palatinizada e, além disso, havia expectativa de confecção de prótese dental removível para reabilitar as perdas dentais superiores, sendo necessária a preservação do fundo de vestíbulo superior direito. Foi realizada uma incisão palatina pediculada com lâmina fria número $15 \mathrm{com}$ aproximadamente $10 \mathrm{~mm}$ de largura, $15 \mathrm{~mm}$ de 
Research, Society and Development, v. 11, n. 1, e35311124538, 2022

(CC BY 4.0) | ISSN 2525-3409 | DOI: http://dx.doi.org/10.33448/rsd-v11i1.24538

comprimento (Figura 3). A incisão respeitou 3mm de distância da margem do dente canino e incisivo lateral direitos. O retalho dividido foi remanejado para a região do alvéolo e suturado com sutura simples e uso de fio de nylon (Figura 4 e 5). O periósteo foi mantido aderido ao osso na região cruenta.

Figura 3. Delineamento do retalho palatino para o fechamento da comunicação bucossinusal.

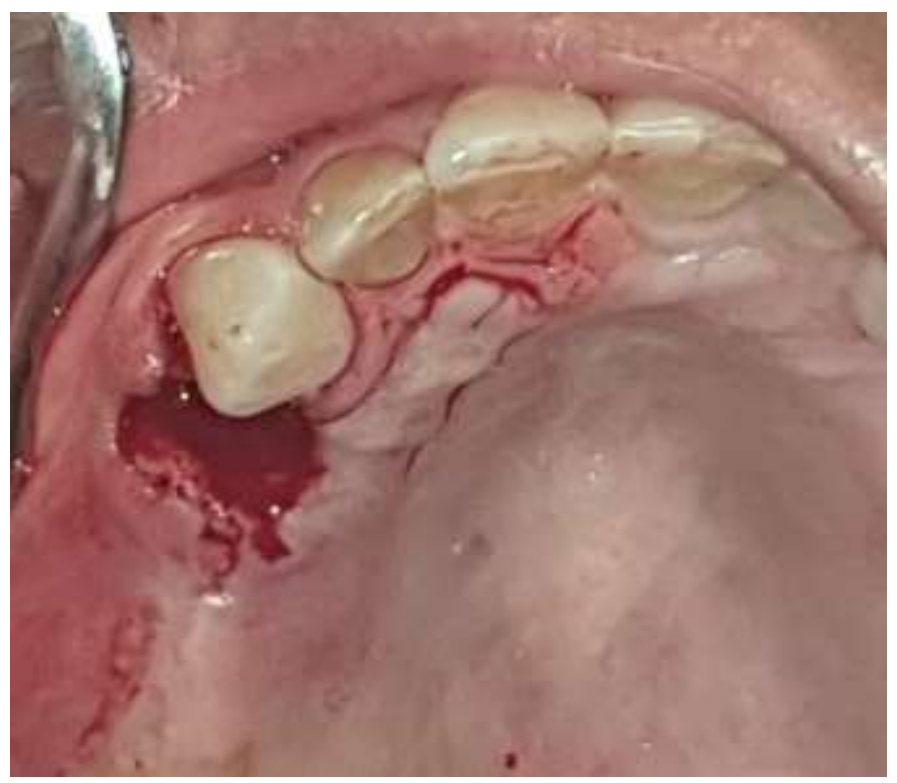

Fonte: Autores (2022).

Figura 4. Remanejamento do retalho para a região cirúrgica.

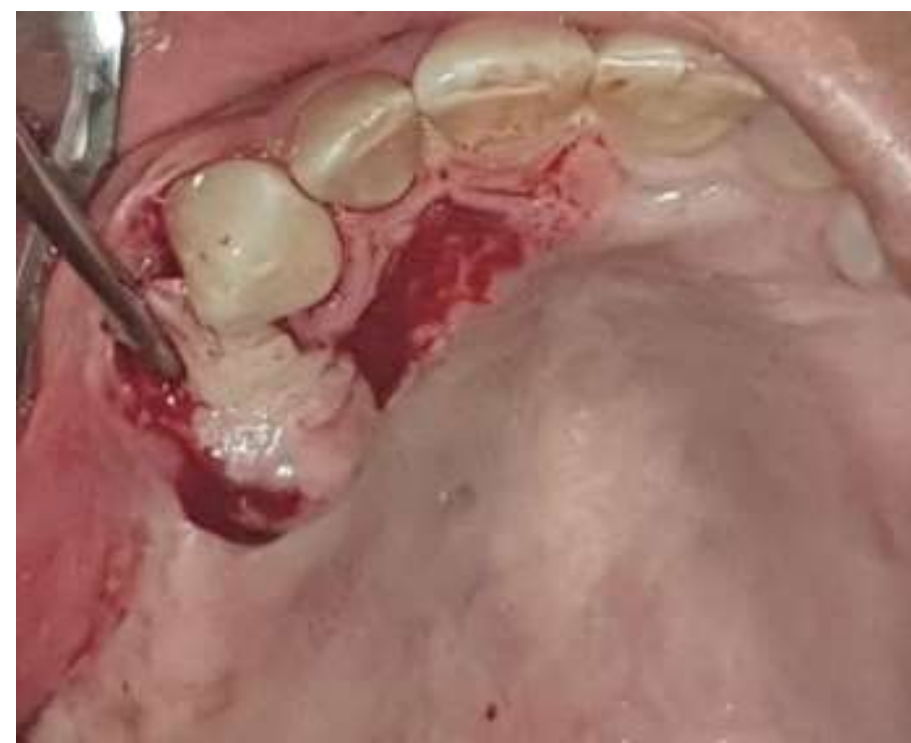

Fonte: Autores (2022). 
Figura 5. Loja cirúrgica ao final do procedimento.

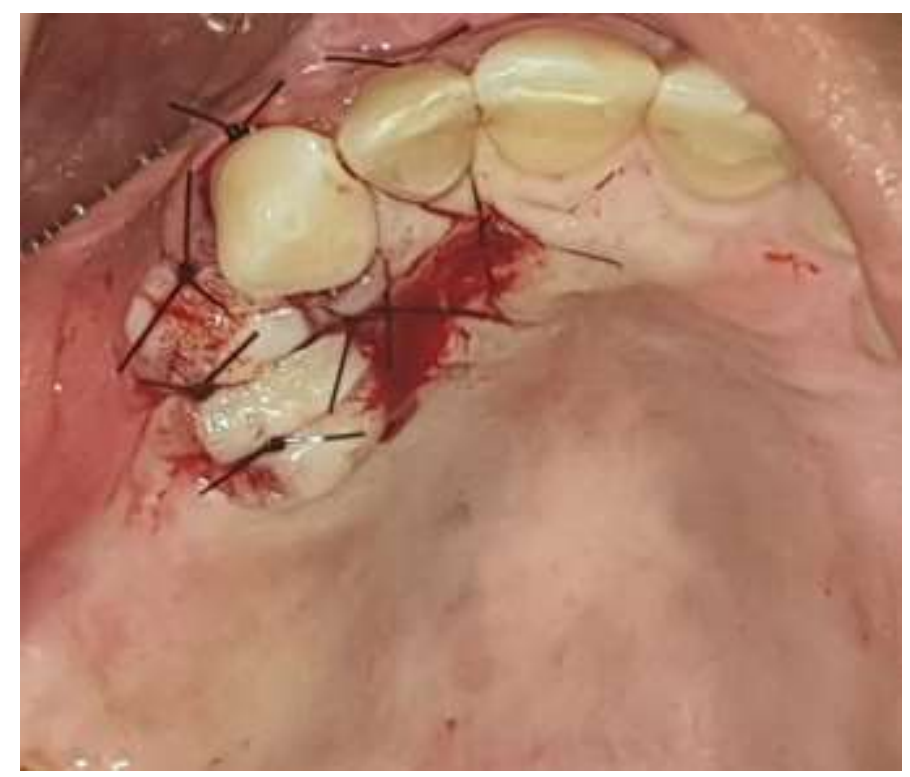

Fonte: Autores (2022).

O paciente foi orientado no pós-operatório, em resumo, a não assoar o nariz por 14 dias, abrir a boca para espirar e tossir, ter uma dieta liquida ou pastosa por 3 dias e evitar atividades físicas por 3 dias. Foi prescrito: amoxicilina associada a clavulanato de potássio $(500 \mathrm{mg}+125 \mathrm{mg})$ de 8 em 8 horas por 7 dias, nimesulida 100mg de 12 em 12 horas por 3 dias e dipirona sódica 500mg de 6 em 6 horas por 3 dias. Ademais, foi orientado a retornar após 7 e 15 dias para nova avaliação clínica. Nas consultas de retorno, observou-se boa cicatrização da região doadora e receptora, sem intercorrências (Figura 6).

Figura 6. Pós-operatório de 15 dias.

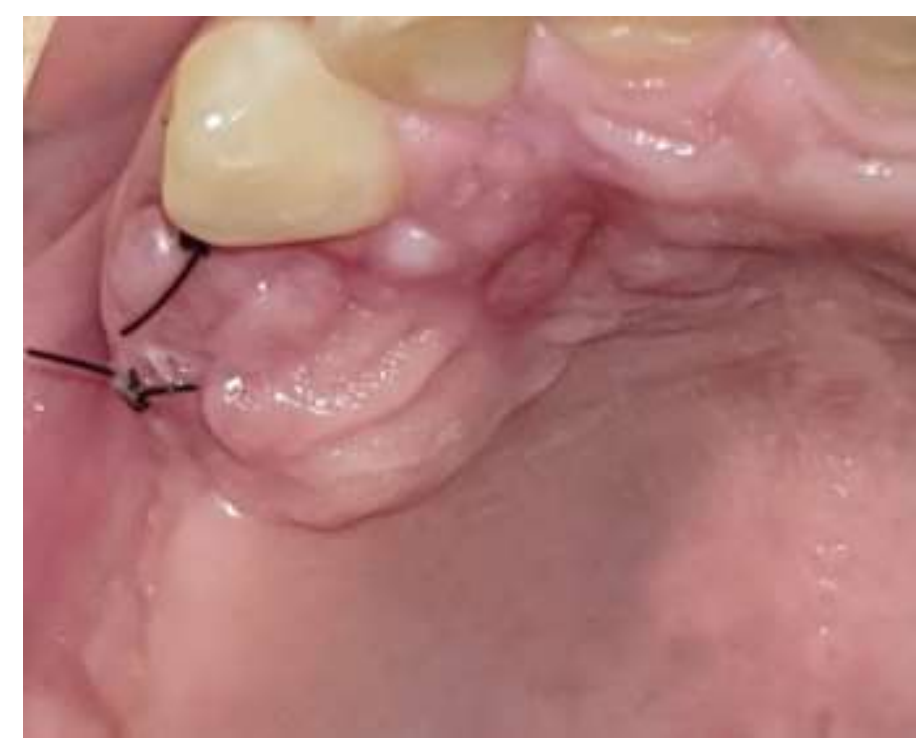

Fonte: Autores (2022).

O paciente foi acompanhado por 63 dias após o procedimento cirúrgico, onde foi possível evidenciar o fechamento completo da comunicação bucossinusal após o reparo dos tecidos moles utilizados no retalho palatino (Figura 7). Com a utilização do retalho palatino, o fundo de vestíbulo superior direito foi preservado, não havendo nenhuma interferência para a confecção e instalação da prótese parcial removível (Figura 8). 
Figura 7. Pós-Operatório de 63 dias, onde é possível constatar a adequada cicatrização do sítio cirúrgico e a normalidade dos tecidos na região operada.

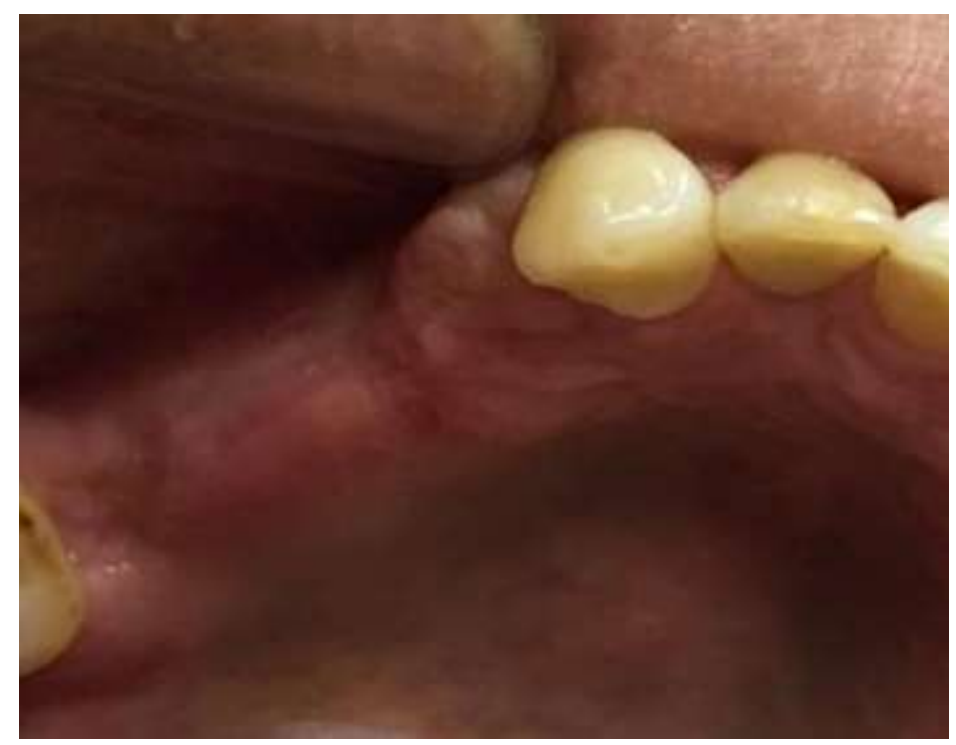

Fonte: Autores (2022).

Figura 8. Paciente com a prótese parcial removível superior, sem interferências na adaptação da prótese.

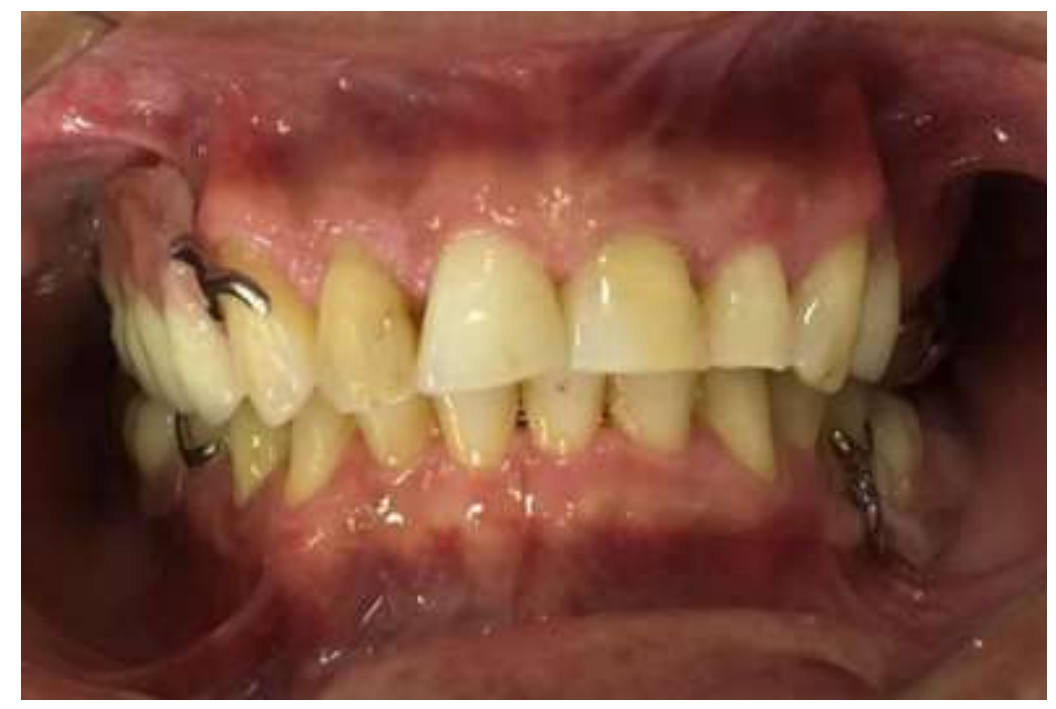

Fonte: Autores (2022).

\section{Discussão}

A comunicação bucossinusal é uma intercorrência operatória caracterizada pela existência de uma comunicação entre a cavidade oral e o seio maxilar, ocasionada pela perda dos tecidos moles e duros que separam ambos os compartimentos. Esse acidente ocorre mais comumente durante a extração de dentes molares superiores e pré-molares (48\%). A principal razão é a proximidade anatômica ou projeção das raízes em relação ao seio maxilar (Hassan et al., 2012). O diagnóstico é geralmente baseado na manobra de Valsalva, que oferece uma sensibilidade de 52\%, além da inspeção visual, da palpação alveolar, da análise dos exames por imagem e dos aspectos subjetivos do paciente (Ehrl,1980). A tomada de decisão clínica para o fechamento da comunicação bucossinusal envolve vários fatores como o tamanho e posição da comunicação, o tempo de diagnóstico, a presença de infecção, a quantidade e qualidade dos tecidos disponíveis, a possibilidade de realização de tratamentos reabilitadores e a experiência do profissional (Dym \& Wolf, 2012) (Amorim et al. 2020), (Macedo et al. 2020). 
Embora a prevalência de comunicações bucossinusais em molares (30\% de casos em terceiro molar, $45 \%$ em segundo molar e 27,2\% em primeiros molares) seja maior que em pré-molares (5,3\%), a incidência desse tipo comunicação envolvendo esses dentes (pré-molares) ainda é controversa, sobretudo, em relação aos primeiros pré-molares (Parvini et al., 2019). De acordo com o estudo proposto por Narendar et al. (2017), não foi relatada comunicação oroantral envolvendo pré-molares, oposto que, segundo a avaliação feita por Batra et al. (2010), cerca de 5,2\% dos casos de comunicação bucossinusal foram de primeiro pré-molar e 15,7\% foram de segundo pré-molar (Batra et al., 2010).

Diversas técnicas são propostas para o manejo das comunicações bucossinusais, como por exemplo, a rotação do retalho palatino, o deslizamento do retalho vestibular e a manipulação do corpo adiposo da bochecha (Amorim et al. 2020), (Macedo et al. 2020). A escolha de qual manobra utilizar varia dependendo das características do defeito, da sua localização e da habilidade do cirurgião. No contexto clínico em questão, por ser um defeito maior que dois milímetros, a abertura não fecha espontaneamente, o que requer o fechamento por meio de técnica cirúrgica (Hanzawa et al., 1995). A técnica utilizada foi a rotação do retalho palatino, justificada por ser viável em região de pré-molar e, ainda, uma boa indicação devido a necessidade de reabilitação protética do paciente (Parvini et al., 2018), pois o reposicionamento dos tecidos não interfere na adaptação da prótese. As vantagens do retalho palatino incluem alta vascularidade, espessura adequada e qualidade do tecido. As desvantagens incluem a necrose do retalho que pode ocorrer devido à excessiva rotação do retalho (Khandelwal \& Hajira, 2017), exposição da superfície óssea, sintomatologia dolorosa e irregularidade da superfície tecidual devido a epitelização secundária no pós-operatório (Borgonovo et al., 2012). No presente caso clínico, no entanto, a opção por manter o periósteo aderido ao osso na região cruenta foi importante para minimizar essas desvantagens, visto que a manutenção do periósteo deve ser considerada para a obtenção de melhores efeitos terapêuticos (Lin et al. 2014), e consequentemente oferecer ao paciente um pós-operatório mais confortável.

Do ponto de vista ético e legal, sabe-se que as comunicações bucossinusais são acidentes, até certo ponto, previsíveis e nem sempre evitáveis. Cabe ao profissional, portanto, alertar e esclarecer adequadamente o paciente sobre os riscos inerentes às extrações de dentes superiores e, caso a chance seja alta de comunicação bucossinusal em determinado caso, que as opções terapêuticas e custos adicionais sejam discutidos. Neste contexto, a aplicação de um Termo de Consentimento Livre e Esclarecido ao paciente é a conduta esperada e adequada com o objetivo de resguardar as partes quanto à correta informação prestada, satisfazendo aspectos éticos, bioéticos e legais.

\section{Conclusão}

A comunicação bucossinusal decorrente de extração de primeiro pré-molar superior é um acidente atípico e com baixa incidência, quando comparado com os demais dentes superiores posteriores. Diante desta intercorrência há necessidade de abordagem profissional acurada, iniciando pelo diagnóstico precoce, planejamento, seleção e execução de técnica de fechamento adequados, além do devido acompanhamento clínico e colaboração do paciente até o fechamento total da comunicação. O presente caso mostra que a rotação de retalho palatino é um método seguro e capaz de promover resultados satisfatórios, além de abrir espaço para a publicação de artigos futuros e, consequentemente, promover uma nova discussão sobre a prevalência desses acidentes na região de pré-molares.

\section{Referências}

Amorim, A. V. B. A., Souza, J. A. N., Júnior, F. A. S., Brito, P. H., Laffitte, C. M., Lemos, E. A., Crispim, L. S., Rocha, S. O. A., Pereira, G. M. A., Junior, F. A. S. (2020). Closure of buccosinusal communication with bichat ball: case report. Research, Society and Development, [S. l.], v. 9, n. 12.

Batista, P., Franco, A., \& Wichnieski, C. (2011). Contribuição para o estudo do seio maxilar. Revista Portuguesa de Estomatologia, Medicina Dentária e Cirurgia Maxilofacial, 52(4), 235-239. 
Batra, H., Jindal, G., \& KAUR, S. (2010). Evaluation of different treatment modalities for closure of oro-antral communications and formulation of a rational approach. J Maxillofac Oral Surg. v. 9, p. 8-13.

Borgonovo, A.E., Berardinelli, F.V., Favale, M., \& Maiorana, C. (2012). Surgical Options in Oro-antral Fistula Treatment. Open Dent J, v. 6, p. 94-98, 2012.

Camarini, T. E., Kamei, N. C., Farah, G. J., Daniel, A. N., Jacob, R. J., \& Bento, L. A. (2008). Utilização do corpo adiposo bucal para fechamento de comunicação bucosinusal associado à enucleação de cisto residual-relato de caso. Revista de cirurgia e traumatologia buco-maxilo-facial, 7(3), 23-30.

Cardoso, R. F., \& Capella, L. R. C. (2002) Levantamento de seio maxilar. Odontologia. Periodontia, cirurgia para implantes, cirurgia, anestesiologia.

Del Junco, R., Rappaport, I., \& Allison, G. R. (1988). Persistent oral antral fistulas. Archives of otolaryngology--head \& neck surgery, 114(11), $1315-1316$.

Dym, H. \& Wolf, JC. (2012). Oroantral communication. Oral Maxillofac Surg Clin North Am. v. 24, p. 239-247.

Ehrl, P. A. (1980). Oroantral communication. International journal of oral surgery, v. 9, p. 351-358.

Hassan, O., Shoukry, T., Raouf, A.A. \& Wahba, H. (2012). Combined palatal and buccal flaps in oroantral fistula repair. Egypt Journal of Ear, v. 13, p. 77-81.

Hanzawa, Y., Itoh, K., Mabashi, T., \& SATO, K. (1995). Closure of Oro-antral Communication Using a Pedicle Buccal Fat Pad Graft. J Oral Maxillofac Surg, v. 53, p. $771-775$

Hupp, J. R., Ellis, E., \& Tucker, M. R. (2008). Contemporary oral and maxillofacial surgery. St. Louis, Mo: Mosby Elsevier.

Hupp, J. R., Ellis, E., \& Tucker, M. R. (2019). Contemporary oral and maxillofacial surgery (Seventh edition.). St. Louis: Elsevier.

Haas, R., Watzak, G., Baron, M., Tepper, G., Mailath, G., \& Watzek, G. (2003). A preliminary study of monocortical bone grafts for oroantral fistula closure. Oral surgery, oral medicine, oral pathology, oral radiology, and endodontics, 96(3), 263-266.

Khandelwal, P., \& Hajira, N. (2017). Management of Oro-antral Communication and Fistula: Various Surgical Options. World J Plast Surg, v. 6, p. 3-8.

Lin, Z., Fateh, A., Salem, D. M., \& Intini, G. (2014). Periosteum: biology and applications in craniofacial bone regeneration. Journal of dental research, 93(2), $109-116$.

Macedo, R. A. P., Pereira, V. B. S., Barros, A. V. M., Rodrigues, E. D. R., Santos, K. R., Vasconcelos, B. C. E., Barbirato, D. S. (2020) Surgical closure of oroantral communication with L-PRF: a case report. Research, Society and Development, [S. l.], v. 9, n. 10.

Miloro, M., Ghali, G., Larsen, P., \& Waite, P. (2012). Peterson's Principles of Oral and Maxillofacial Surgery. Third Edition.

Narendar, R., Balakrishnan, G., Kavin, T., Venkataraman, S., Altaf, S. K., \& Gokulanathan, S. (2017). Incidence of Risk and Complications Associated with Orthodontic Therapeutic Extraction. J Pharm Bioallied Sci. v. 9, p. 201-204.

Palhano, J. M. L., Cordeiro, A. A., Gregório, B. A., Dias, B. A. S., Sousa, F. R. G., Gregório, J. A., Figueiredo, L. L. M., Deus, L. A. A., Silva, M. A. P., Silva, M. I. A. A., Rodrigues, M. A., Veríssimo, M. H. G., Paiva, P. R. S., Pereira, P. A. A., Lima, R. R. (2020) Closure of oroantral fistula using bichat adipose body: narrative literature review. Research, Society and Development. [S. l.], v. 9, n. 12.

Parvini, P., Obreja, K., Begic, A., Schwarz, F., Becker, J., Sader, R., \& Salti, L. (2019). Decision-making in closure of oroantral communication and fistula. International journal of implant dentistry, 5(1), 13.

Parvini, P., Obreja, K., Sader, R., Becker, J., Schwarz, F., \& Salti, L. (2018). Surgical options in oroantral fistula management: a narrative review. Int J Implant Dent. v. 4, p. 40.

Pereira, A. S., Shitsuka D. M., Parreira, F. J., Shitsuka, R. (2018). Metodologia da pesquisa científica. [e-book]. Santa Maria. Ed. UAB/NTE/UFSM.

Rodrigues, M. T. V., Schueng, F. E. A., Mendes, B. C., Macedo, F. G. C., Nunes, F., Noia, C. F. (2021). Intra-sinus complex odontoma management simultaneously to oroantral communication closure: Why remove it?. Research, Society and Development, [S. l.], v. 10, n. 6.

Scattarella, A., Ballini, A., Grassi, F. R., Carbonara, A., Ciccolella, F., Dituri, A., Nardi, G. M., Cantore, S., \& Pettini, F. (2010). Treatment of oroantral fistula with autologous bone graft and application of a non-reabsorbable membrane. International journal of medical sciences, 7(5), 267-271.

Yih, W. Y., Merrill, R. G., \& Howerton, D. W. (1988). Secondary closure of oroantral and oronasal fistulas: a modification of existing techniques. Journal of oral and maxillofacial surgery: official journal of the American Association of Oral and Maxillofacial Surgeons, 46(5), 357-364. 